\title{
Evaluation of LiDAR data processing at the mobile network edge for connected vehicles
}

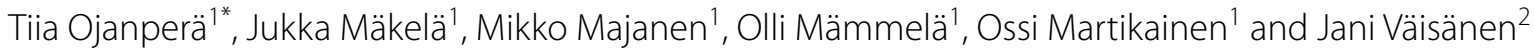

\author{
*Correspondence: \\ tiia.ojanpera@vtt.fi \\ ${ }^{1}$ VTT Technical Research \\ Centre of Finland Ltd., \\ Kaitoväylä 1, 90571 Oulu, \\ Finland \\ Full list of author information \\ is available at the end of the \\ article
}

\begin{abstract}
5G mobile network technology together with edge computing will create new opportunities for developing novel road safety services in order to better support connected and automated driving in challenging situations. This paper studies the feasibility and benefits of localized mobile network edge applications for supporting vehicles in diverse conditions. We study a particular scenario, where vehicle sensor data processing, required by road safety services, is installed into the mobile network edge in order to extend the electronic horizon of the sensors carried by other vehicles. Specifically, we focus on a LiDAR data-based obstacle warning case where vehicles receive obstacle warnings from the mobile network edge. The proposed solution is based on a generic system architecture. In this paper, we first evaluate different connectivity and computing options associated with such a system using ns-3 simulations. Then, we introduce a proof-of-concept implementation of the LiDAR-based obstacle warning scenario together with first results from an experimental evaluation, conducted both in a real vehicle testbed environment and in a laboratory setting. As a result, we obtain first insights on the feasibility of the overall solution and further enhancements needed.
\end{abstract}

Keywords: 5G, Automotive vertical, MEC, LiDAR, Experimental evaluation, Testbed, Simulations

\section{Introduction}

The current technological transformation, taking place in the automotive industry, in terms of vehicles getting more and more connected to each other and to the cloud, is foreseen to improve the safety and efficiency of roads. Digital services, offered to vehicle drivers in real time, with the purpose of preventing traffic accidents and avoiding casualties, are essential in realizing the benefits envisioned. Especially, accurate and focused road weather and road condition information plays a key role here, together with direct incident and control information. Furthermore, similar services will pave the way for full automation of vehicles in the future as they can help extending the electronic horizon of the vehicles beyond their own sensors.

In advanced road weather and safety services, data from fixed roadside sensors combined with extensive observation data from vehicles are used to increase author(s) and the source, provide a link to the Creative Commons licence, and indicate if changes were made. The images or other third party material in this article are included in the article's Creative Commons licence, unless indicated otherwise in a credit line to the material. If material is not included in the article's Creative Commons licence and your intended use is not permitted by statutory regulation or exceeds the permitted use, you will need to obtain permission directly from the copyright holder. To view a copy of this licence, visit http:// creativecommons.org/licenses/by/4.0/. 
the accuracy and coverage of the information and alerts produced [1]. So far, the lack of sufficient communication media has hindered the wide-scale adoption of such services, however. 5G together with edge computing (e.g., ETSI multi-access edge computing (MEC)) [2,3] is expected to create new possibilities, particularly for the vehicular sensor and video data communication and processing, required by the advanced road weather and safety services. The increased transmission capacity, especially in the uplink direction, and lower latency of 5G will enable vehicular services to utilize and build intelligence upon data collected from vehicles traveling on the roads, in real time. The data in question can be produced by a variety of sensors and systems installed on the vehicles, including LiDARs, radars, cameras, and friction sensors [4]. In addition, MEC will enable bringing the data processing and cloud-based services physically closer to the vehicles, thus allowing offloading the burden of data processing from vehicles to the edge cloud, if needed, as well as collaborative sensing using data from multiple vehicles.

In the future, cars will be close to the level of small-scale supercomputers when it comes to their data processing capabilities. Yet, having additional data processing capacity and algorithms running in the network entities, such as MEC servers, has its own incentives. Firstly, computing in vehicles always comes with a cost. Thus, we cannot assume that all vehicles (cf. non-premium models) will have limitless computing capacity to serve all applications even in the future. Secondly, a networkcentered approach may be lucrative in some cases in the business perspective as well, thanks to easier software updates and better security and integrity. In such an approach, no other access to the vehicles themselves is needed besides the sensor data collection and feeding back the processed information and warnings. Finally, the approach allows collecting and fusing data from multiple vehicles and using the same data for the purposes of different applications running in the edge.

This paper presents an initial study on the practical feasibility of a 5G-inspired MEC application in providing real-time warnings based on vehicle observations. We focus on the case of LiDAR-based obstacle detection and describe the overall scenario, architecture and proof-of-concept implementation of the solution. The prototype is evaluated both in a real vehicular environment and in a laboratory setting. This paper is partly based on the results presented in the paper [5], yet extended with additional details and findings from a simulation study. As a result, we obtain both simulated and practical experiences from using a MEC application to generate road condition warnings based on vehicle observations. We also identify places for future enhancements in order to make the overall concept a reality.

The rest of the paper is organized as follows: Section 2 summarizes the research methods used in the paper. Section 3 gives an overview of the related work. Section 4 introduces our proposed system architecture and scenario. Section 5 presents the simulation study and analysis of the results. Sections 6 and 7 describe the proofof-concept implementation of the LiDAR-based obstacle warning scenario and the experimental results obtained using the prototype, respectively. Finally, Sects. 8 and 9 provide the discussion and conclusions of the paper as well as an outlook for future work. 


\section{Methods}

This paper presents both simulated and experimental results obtained in the context of the 5G-inspired road safety use cases and the corresponding system architecture, considered in the paper. The simulation study is conducted using ns-3-the discrete-event network simulator for Internet systems. The experimental results are produced in a real field testing environment, including vehicles, vehicular sensors and a $5 \mathrm{G}$ test network, and using a proof-of-concept implementation of a 5G-assisted automated driving scenario. The experimental results are also reproduced in a more controlled laboratory setting for comparison. Due to the differences in the communication technologies and capabilities supported by the simulator and testbed environments, the results are presented separately for each in their corresponding sections.

\section{Related work}

Intelligent transport systems (ITSs) and underlying vehicle-to-vehicle (V2V) and vehicle-to-infrastructure (V2I) communication have traditionally been implemented using wireless local area network connectivity, based on the IEEE 802.11p standard [6]. IEEE 802.11p WAVE/DSRC is the standard used in US for V2V communication, and ITS-G5 is the European version. They are both based on the 802.11p, but they have differences on the upper MAC layer and above. A major drawback of 802.11 -based systems is the cost of the roadside infrastructure, required by the V2I services, which has severely limited the adoption of the technology. Recently, the adoption of DSCR has faced further challenges in the US due to FCC's decision to reallocate the spectrum reserved for DSRC to other uses in November 2020. Nevertheless, Europe continues to promote a hybrid approach supporting ITS-G5.

For vehicle-to-network (V2N) communication, 3GPP-standardized 5G technology [2] offers a complementary solution to $802.11 \mathrm{p}$ without the need for a dedicated network infrastructure for automotive vertical services. Instead, it is envisioned that even critical services can be delivered through a public or shared 5G network infrastructure, thanks to emerging capabilities such as network slicing. Furthermore, 5G will enable novel ITS services requiring a higher bandwidth for data transmission and more flexibility in the delivered content than what is offered by 802.11p and the associated US and European ITS standards.

For V2V and V2I, 3GPP's cellular V2X (C-V2X) communication facilitates direct communication between vehicles and roadside infrastructure, thus offering an alternative solution to 802.11p. LTE device-to-device (D2D), also referred to as sidelink (SL), was introduced in the LTE Release 12. It has two modes: in Mode 1 the eNB manages the direct communication scheduling between UEs, while in Mode 2 the UEs manage the scheduling independently from the eNB. Thus, in Mode 2 the UEs do not need to be in the coverage area of any $\mathrm{eNB}$, which makes this mode more suitable for vehicular safety applications. In LTE Release 14, additional modes for D2D communication were specified, especially for vehicular scenarios. Like Mode 1, also Mode 3 is based on eNB for managing the $\mathrm{V} 2 \mathrm{~V}$ communication, while Mode 4 is similar to Mode 2 and does not depend on the eNB. Thus, the Mode 4 should be the best solution for vehicular scenarios requiring direct V2V communication. The difference between Modes 1 \& 2 and Modes 
$3 \& 4$ is that Modes $1 \& 2$ were designed with battery consumption as a priority, while Modes 3 \& 4 were designed especially for V2V communication with latency, reliability and scalability in mind. Release 16 specifies the 5G NR-based sidelink communication for V2X. The upcoming Release 17 in 2022 will include enhancements to the 5G NRV2X communication, e.g., sidelink relay and discontinuous reception mode.

The emergence of $5 \mathrm{G}$ and Internet of Things (IoT) type of communications has risen the interest towards edge computing and solutions such as MEC [3, 7]. In the MEC paradigm, mobile computing, network control and storage are pushed to the edge of networks, that is, close to the access points. By doing so, MEC can enable computation-intensive and latency-critical applications at mobile devices, which are typically resource-limited. In many scenarios, MEC is seen as a point for hosting applications, but it may provide also computing resources for AI-guided network and radio resource management, for example for radio access technology (RAT) selection for vehicular communication [8]. For $5 \mathrm{G}$ automotive vertical services, MEC is also a key enabler for low-latency communications and distributed sensor data collection and processing.

Regarding MEC and automated driving, a few research papers are available in the literature [9-12]. Ogawa et al. reported field experiments with sensor data transmission over the $5 \mathrm{G}$ network [13]. Industry trials have also taken place to evaluate the capabilities of cellular technology to enable low-latency car connectivity [14]. To the best of our knowledge, this paper is the one of the first works detailing the feasibility and benefits of localized MEC applications both in a real vehicle testbed environment and in a laboratory setting.

For sensor and IoT data communication, a number of different protocols and solutions exist. In this work, we consider Apache Kafka [15] and Message Queuing Telemetry Transport (MQTT) [16] protocols. Both are lightweight publish-subscribe messaging transport protocols that function on top of TCP/IP. By using TCP/IP-based protocols, we are able to avoid any problems related to the network firewalls and Network Address Translation (NAT) in the used test networks. Performance-wise, a UDP-based solution, such as Constrained Application Protocol (CoAP) [17], may be better as indicated by our earlier study [1], but adding support for it in the test networks is left for future work.

\section{Scenario for 5G-assisted automated driving}

In our prior work, we have proposed a generic system architecture for 5G-enabled road safety services [1]. The architecture is based on three use cases, namely Use Case 1: "Local focused road weather and safety services", Use Case 2: "Road safety improvement through real-time vehicular video and LiDAR data", and Use Case 3: "Ultra-low delay services for autonomous driving". The use cases were chosen so that they encompass a variety of road safety services that utilize real-time sensor and video data transmitted from a vehicle to another or from a vehicle to MEC/cloud. For the road weather services, a roadside infrastructure of weather stations is considered as a additional source of information to the vehicle observations. In this paper, we focus on Use Case 3 and the 5G-assisted automated driving scenario outlined in this section. For a detailed design of the services associated with the other use cases as well as experimental results, an interested reader is referred to [18]. 
The ultimate purpose of Use Case 3 is to study and demonstrate how low latency or safety-critical control information can be used for improving the safety of automated driving. The information may include various alerts, such as emergency breaking, challenging weather or obstacles, and other information for vehicle control and route planning, such as dynamic maps with road and traffic status data, for example. In the scope of this paper, we focus on a scenario, aiming to demonstrate the feasibility and benefits of 5G and localized MEC applications in supporting automated driving in diverse conditions. We consider a MEC application that uses real-time sensor data from vehicles, processes it with intelligent algorithms, and generates warnings about the road or weather conditions. That is, the vehicle sensor data processing is installed into the network edge in our approach.

In specific, we use vehicle-generated LiDAR data for detecting obstacles on the road. The detailed scenario considered in this paper includes the following steps:

1. Two vehicles are traveling on a road and they are connected to a MEC server over wireless connection. The leading vehicle, referred to as a LiDAR publisher vehicle in this paper, is equipped with a LiDAR sensor, and it is sending a stream of LiDAR data to a MEC server.

2. The MEC server hosts an obstacle detection algorithm that generates warnings based on the LiDAR data collected from vehicles traveling in the area.

3. When an obstacle is detected, the algorithm issues a warning message about the obstacle on the road. The warning is sent to vehicles traveling in the area.

4. An automated vehicle, traveling behind the LiDAR publisher vehicle, receives the warning and acts accordingly (e.g., goes around the obstacle or brakes).

Figure 1 illustrates a sequence diagram of the communications between the two vehicles and MEC server in the scenario. The LiDAR publisher vehicle sends LiDAR data to the MEC server. These data are processed in the MEC server, and if an obstacle is found, a warning message is sent to the automated vehicle.

In this paper, we focus on two types of obstacles that can be detected with LiDAR, namely (1) a physical obstacle on the road (e.g., a human, animal or large debris) that may cause a collision or (2) a hard ridge of snow formed on the road's surface, hindering

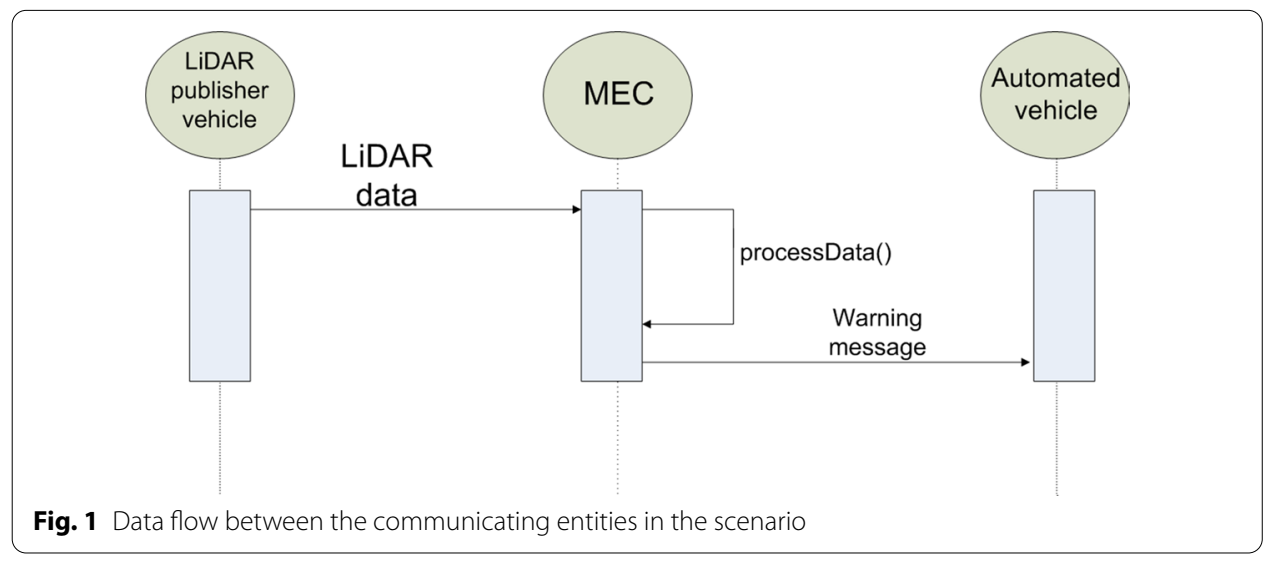


driving and safety, but not blocking the road. Other types of sensor data (e.g., vehicle camera, friction sensors, CAN bus data), sensor fusion and algorithms can be incorporated as well in order to detect more diverse conditions such as potholes or road weather. However, such extensions are left for future work.

\section{Simulation study and results}

Before going into the details of the practical proof-of-concept implementation of our 5 G-assisted automated driving scenario, this section first presents more generic findings related to V2X communication. The results were obtained using ns-3 simulations, and the aim of the simulation study was to gain insights on the impact of the placement of the services and used communication medium to advanced road weather and safety services in the system architecture. The main motivation for the approach is that simulations allow us to test the feasibility of technologies that are not yet available in testbeds. For example, this was the case for LTE V2V at the time of building the prototypes and conducting experiments for this paper. In addition, simulations offer possibilities for testing vehicular communication technologies and solutions in a larger scale than what is possible with test networks and vehicles.

In the remainder of this section, we briefly summarize the simulation scenarios and results obtained. For the purposes of this paper, we selected three key emerging networking topics to be studied in V2X communication scenarios, namely

\section{Multi-Access Edge Computing (MEC) server versus Cloud Computing server \\ 2. $5 \mathrm{G}$ mmWave spectrum versus $4 \mathrm{G}$ LTE radio \\ 3. LTE V2V versus IEEE 802.11p communication.}

In the first case, the end-to-end delay from an UE to the application server was studied. The application server was located either on the remote server in the cloud, connected with a 100-Gbps point-to-point link with 10-ms delay to the packet gateway (PGW) node, or on a MEC server co-located with the eNB. The traffic generated on the UE was constant bit rate UDP traffic, with 10-ms packet interval and 1054 bytes packet size. UE used 4G LTE for the communication. With MEC, the end-to-end delay was $12 \mathrm{~ms}$. With remote server in the cloud, the delay was $22 \mathrm{~ms}$. Since the delay between the eNB and PGW was modeled as $0 \mathrm{~ms}$, the result matches to the point-to-point link delay from PGW to the cloud. We made the same test with using 5G mmWave instead of 4G LTE. With $5 \mathrm{G}$ mmWave, the delay from UE to the MEC decreased to less than $0.5 \mathrm{~ms}$. So, with MEC, the delay caused by the links from the PGW to the destination node can be cut off. Furthermore, 5G mmWave communication technology reduces the delay between UE and eNB. Using both MEC and 5G mmWave instead of remote server in the cloud and 4G LTE, the end-to-end delay can be reduced even 97.5\%. The developed ns-3 MEC model was released for the public, and it is available in [19].

5G mmWave decreases the delay, but it can also provide much more bandwidth, which is essential for transmitting, for example, LiDAR or video data. As an example, we simulated a scenario with many types of vehicular data: LiDAR, video, CAM and DENM messages. The LiDAR data simulated Velodyne HDL-64 LiDAR with packet size of 1206 bytes with 5200 packets/s. The video data packet size was 1400 bytes with 714 packets/s. 


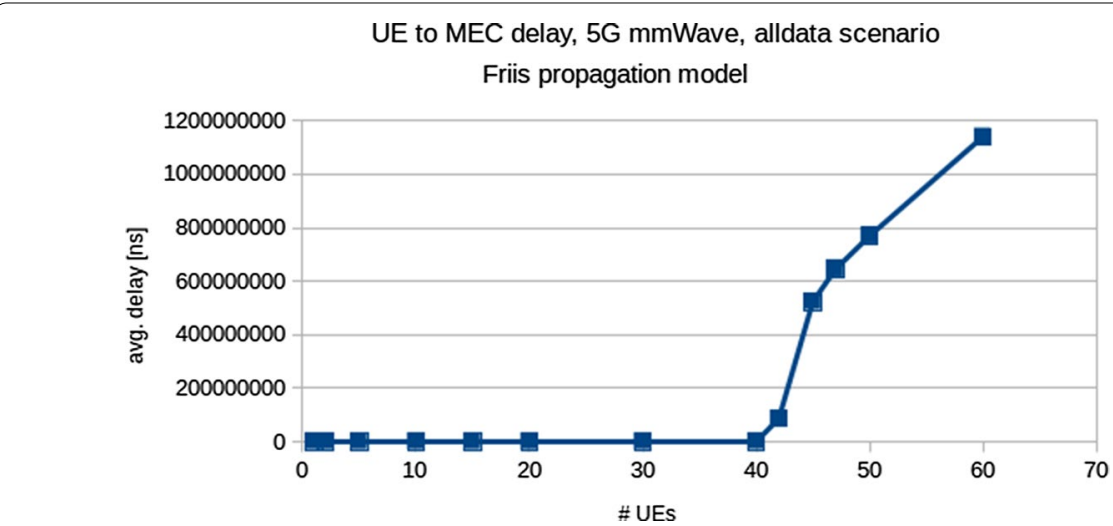

Fig. 2 Average delay from UE to MEC as a function of number of UEs

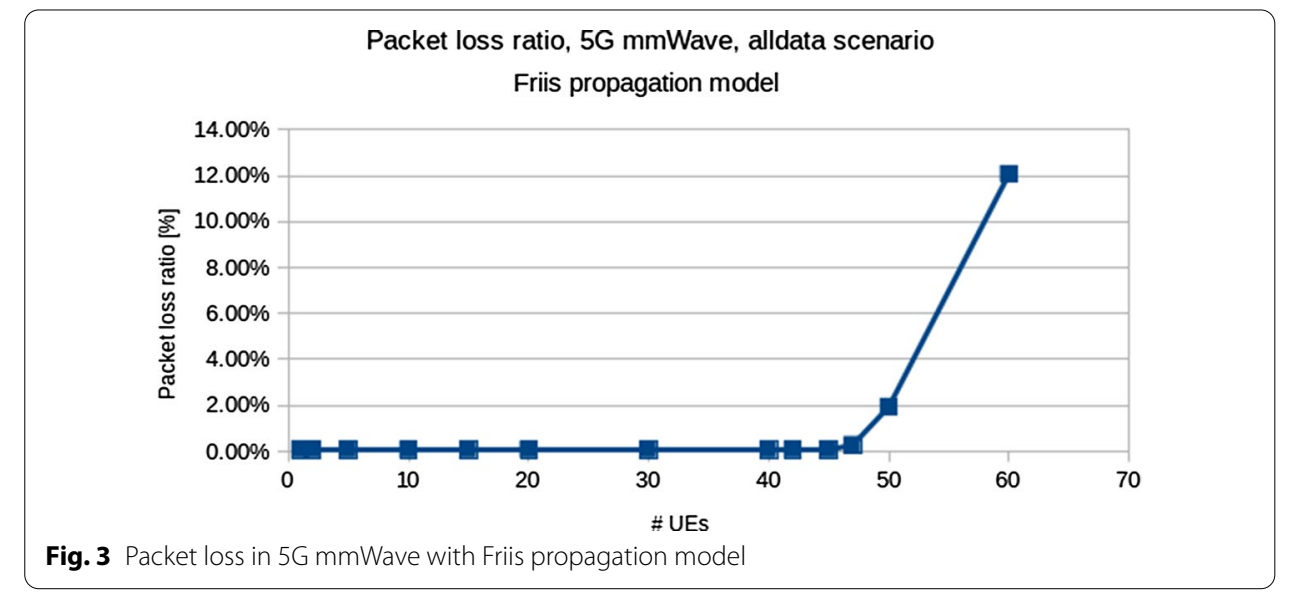

CAM message size was 250 bytes with 10 packets/s. All these data were sent by each UE to the MEC server. DENM message size was 800 bytes, and it was sent by the MEC server to the every UE once per second. Figure 2 represents the average delay for the above-mentioned data packets as a function of number of UEs. The UEs were stationary in a row formation with $10 \mathrm{~m}$ distance from each other. All the UEs were also close enough to the eNB so that there were no packet loss due to the too weak signal strength. (Friis free space propagation model was used.) As can be seen from the figure, the delay remains well below $1 \mathrm{~ms}$ (roughly between 0.3 and $0.6 \mathrm{~ms}$ ) with less than 40 UEs. With more than 40 UEs, the delay increases more rapidly, being over $514 \mathrm{~ms}$ with 45 UEs, for example. Figure 3 shows the packet loss as a function of number of UEs. With 40 UEs or less, the packet loss was constant $0.07 \%$ because of some packets lost in the buffer overflow in the very beginning of the simulation when the communication links were set up. With more than 40 UEs, the packet loss increases. For 4G LTE, the amount of data was way too much even with a single UE. The packet loss even with only one UE was $69 \%$.

We also simulated the same scenario with other propagation and channel models. With 3GPP propagation loss and channel models (provided by the ns-3 mmWave model), the delay and packet loss started to increase already with more than 20 UEs, as depicted in Figs. 4 and 5, respectively. In the 3GPP propagation loss model, some UEs 


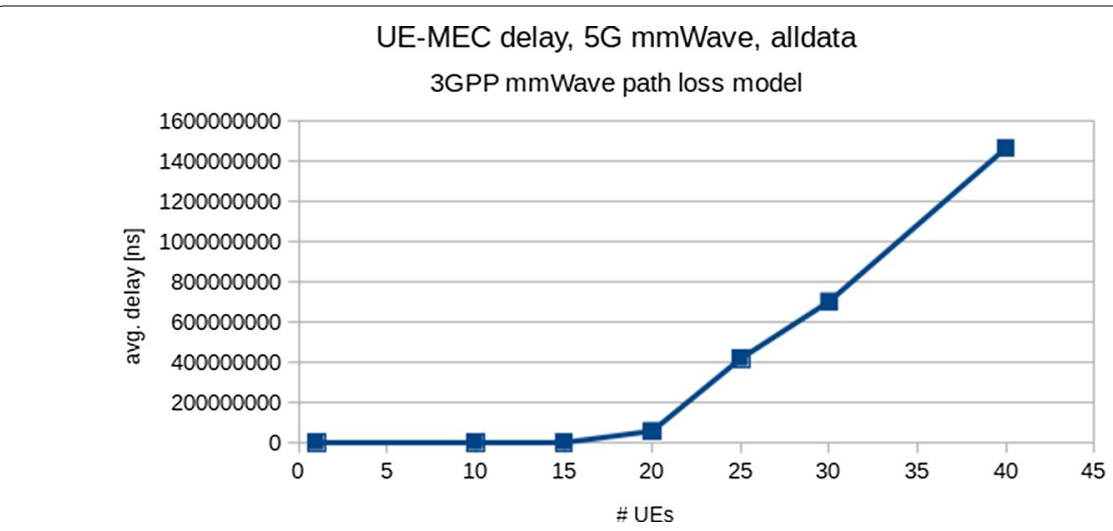

Fig. 4 Delay when 3GPP propagation loss and channel models were used

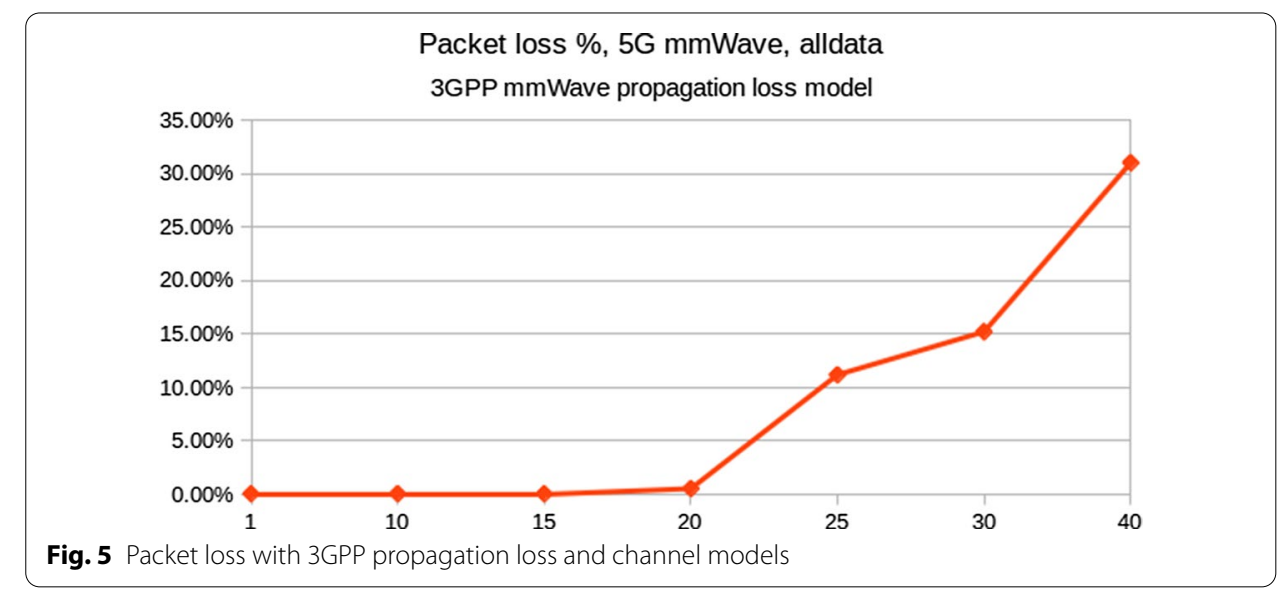

had line of sight (LOS) to the eNB, while others did not, which had effects on the reception of the packets.

We also added some buildings on both sides of the UE queue, that is, to model a street with buildings on both side of the street. In this case, the propagation loss model was set to 3 GPP buildings propagation loss model, also provided by the ns-3 mmWave model. The same 3GPP channel model was used as in the previous simulation. If the eNB was set inside a building, the signal strength was very weak and only the closest UEs were able to communicate. With $1 \mathrm{UE}$, the delay was $1.34 \mathrm{~ms}$ and packet loss $0.07 \%$. With 5 UEs, the delay was already $585 \mathrm{~ms}$ and packet loss $9.29 \%$. However, if the eNB was located on top of the building, the performance was pretty much the same as with the Friis free space propagation model, as depicted in Figs. 6 and 7. The delay increases rapidly with more than 40 UEs, so the real-time communication delay limits of $50 \mathrm{~ms}$ or $100 \mathrm{~ms}$ are exceeded soon after 40 UEs.

In case 3, we studied the delay and packet delivery rate in IEEE 802.11p WAVE/DSRC and LTE D2D mode 2 networks. The ns-3 simulator supports only WAVE/DSRC version and LTE D2D Modes 1 and 2 [20]. Recently, support for Modes 3 and 4 was implemented [21], but unfortunately the model is not publicly available. An analytical model for performance evaluation of Mode 4 has also been developed in [22]. 


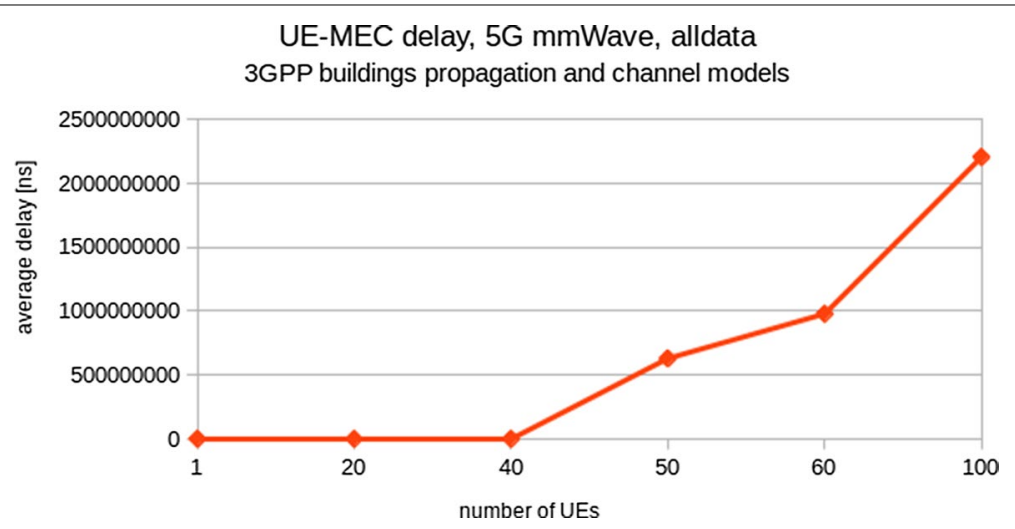

Fig. 6 Delay with 3GPP buildings propagation and channel models, eNB on top of a building

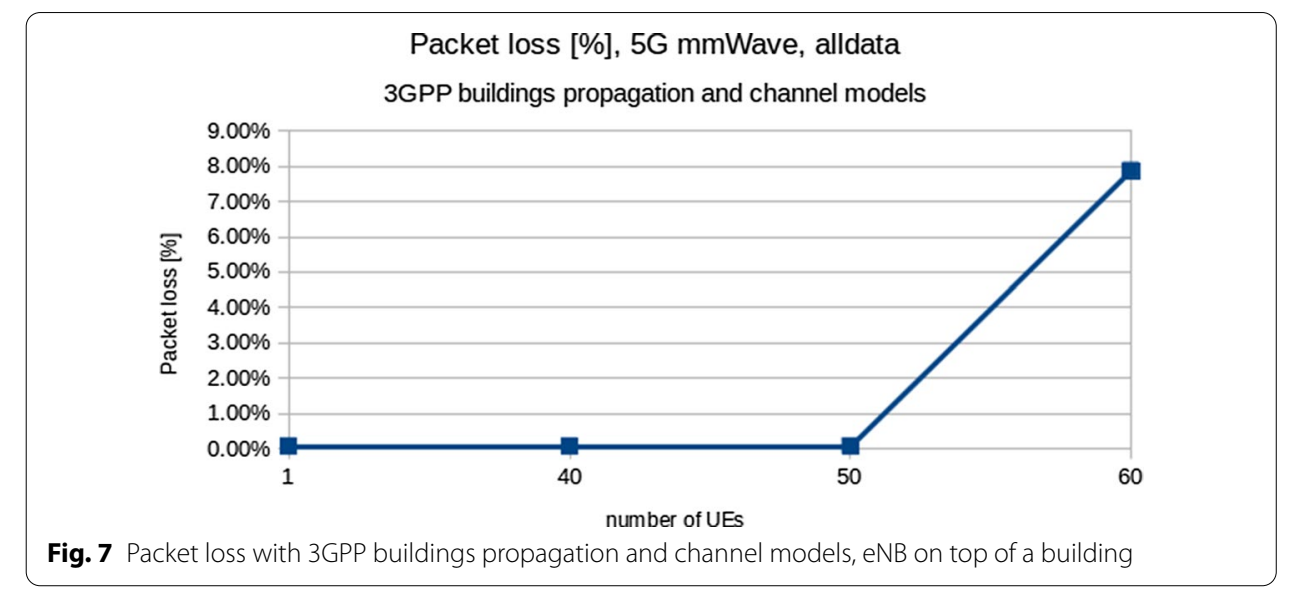

Figures 8, 9, 10, 11, 12 and 13 represent the delay and packet delivery ratio (PDR) with different sizes of CAM messages that are sent by each node every $100 \mathrm{~ms}$. The nodes were aligned in the queue formation $10 \mathrm{~m}$ apart from each other, just like in the previous simulation cases. As can be seen, the average delay is much smaller with 802.11p WAVE/DSRC than with LTE D2D. WAVE/DSRC delay is mainly between 0.5 and $3.5 \mathrm{~ms}$ depending on the CAM message size and number of nodes. Also, PDR is nearly $100 \%$. The small drop with 15 nodes and greater drop with 20 nodes are due to the communication range. The coverage radius with the used simulation settings was about $150 \mathrm{~m}$, i.e., the th node was on the edge of the coverage area of the first node, and 20th node was already out of coverage of the first 1 to 5 nodes in the queue. The physical mode used in 802.11p was OFDM with $6 \mathrm{Mbps}$ data rate and $10 \mathrm{MHz}$ bandwidth. LTE D2D Mode 2 did not suffer from the signal strength-related issues. However, it performed worse than WAVE/DRSC due to the smaller bandwidth. (Only two resource blocks were used.) LTE D2D reached the most stringent delay limit of $50 \mathrm{~ms}$ for real-time communication only with the smallest CAM message size of 100 bytes and with less than 10 UEs. With CAM message size of 800 bytes, it did not even reach the 100-ms delay limit (without optimizing some parameters). Also, with 10 UEs or more, it did not reach the most stringent PDR limit of $95 \%$ with any size of 


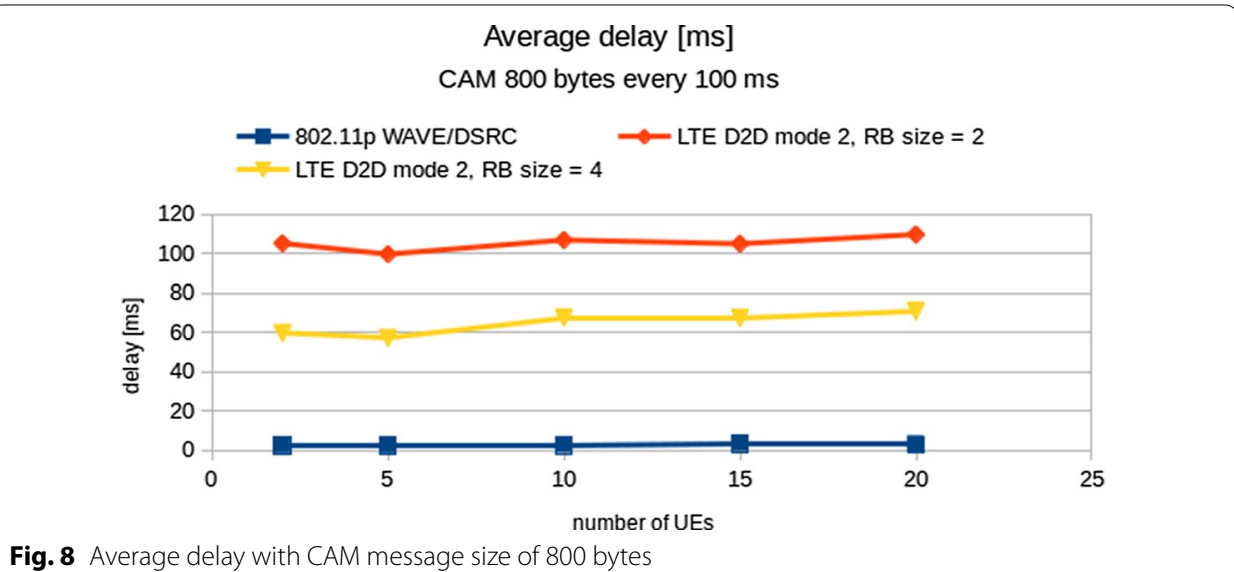

Fig. 8 Average delay with CAM message size of 800 bytes

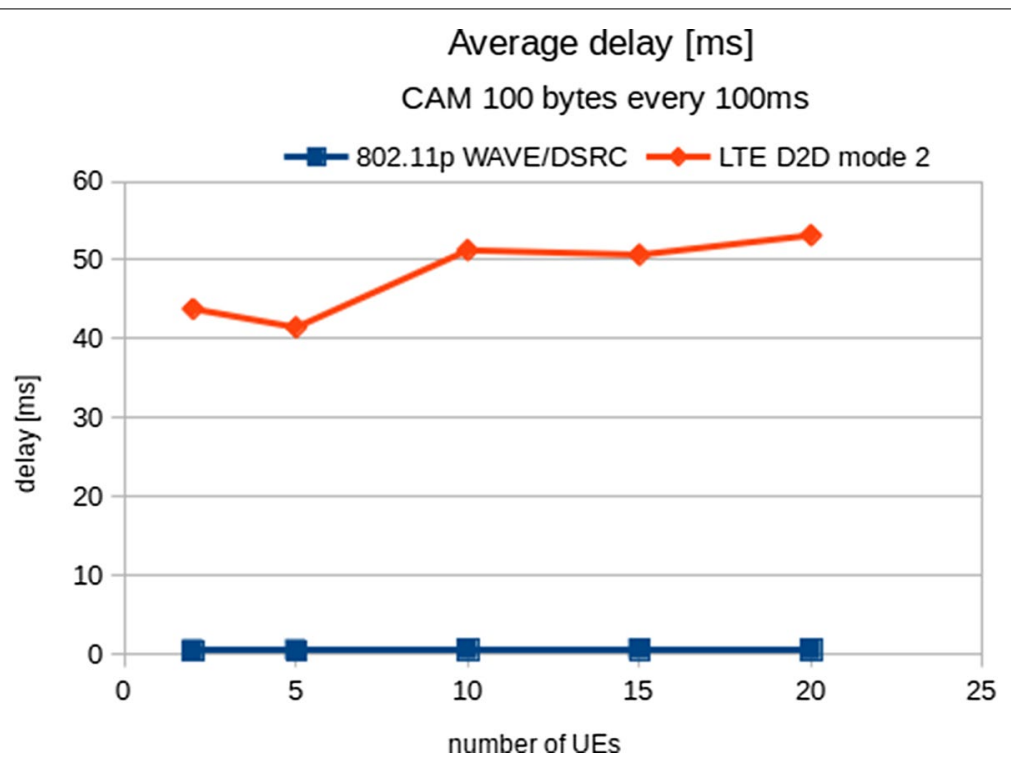

Fig. 9 Average delay with CAM message size of 100 bytes

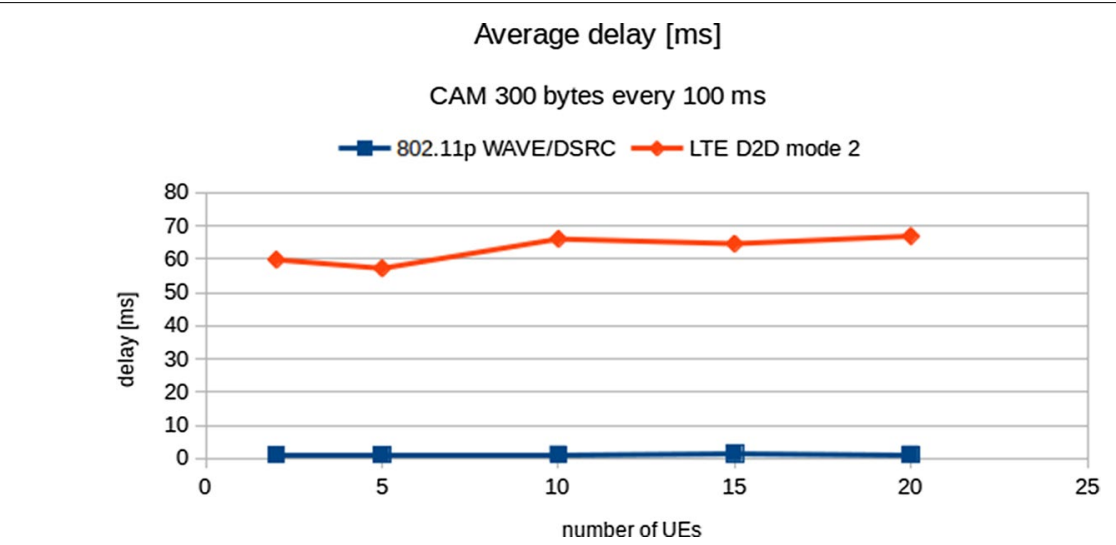

Fig. 10 Average delay with CAM message size of 300 bytes 


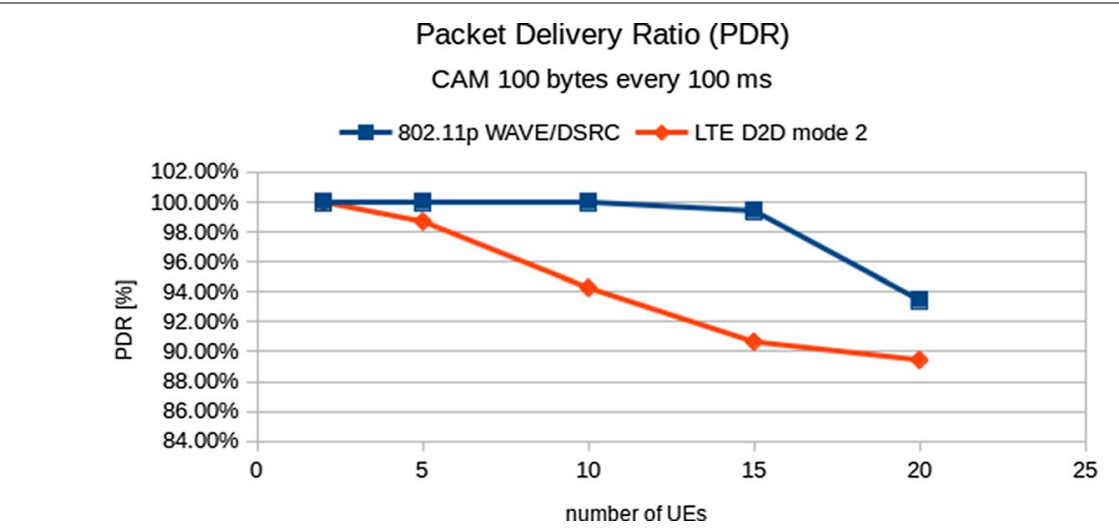

Fig. 11 PDR with CAM message size of 100 bytes

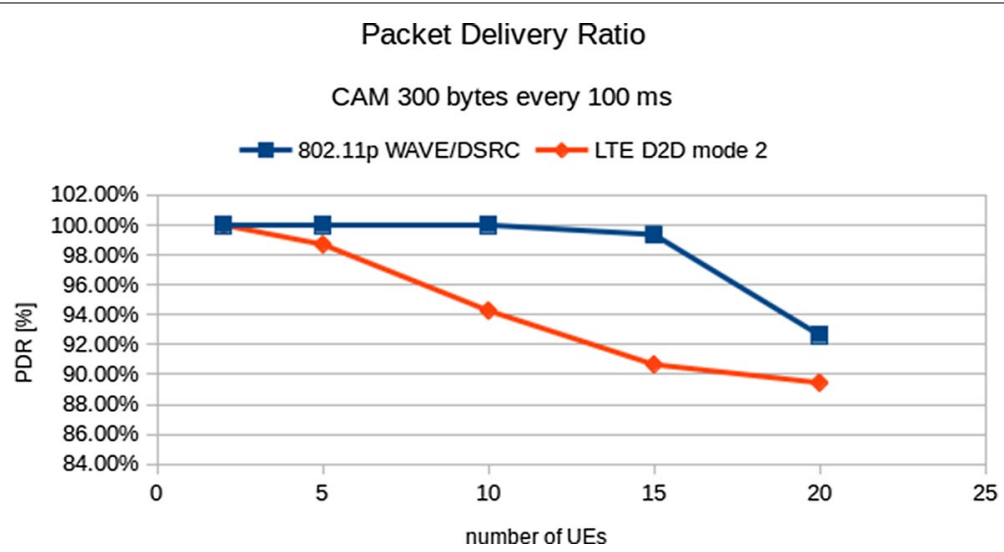

Fig. 12 PDR with CAM message size of 300 bytes

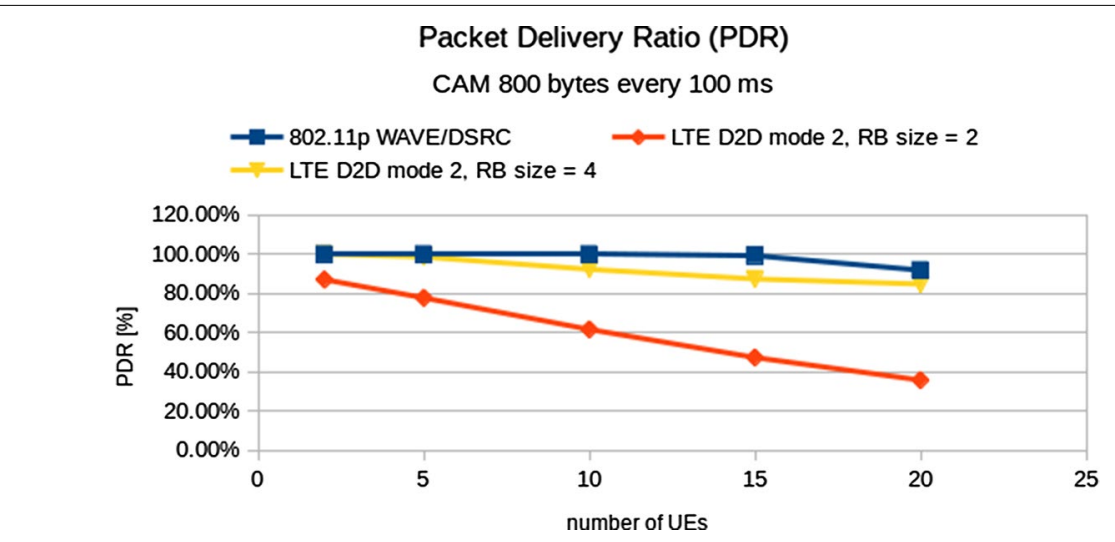

Fig. 13 PDR with CAM message size of 800 bytes

CAM messages. The reasons for poor performance are the smaller amount of bandwidth and the way the sidelink resources are scheduled. There are also several configurable parameters that affect on the performance, and they form over 150 possible 
combinations [23]. It is hard to find the optimal configuration to the current situation. Furthermore, none of the combinations achieves a global optimum [23]. An example is seen in Figs. 8 and 13, when the delay for the increased CAM packet size exceeded $100 \mathrm{~ms}$ and PDR was below $40 \%$ with 20 UEs, but with a small change in resource block (RB) size parameter decreased the delay to 60-70 ms and kept the PDR in over $80 \%$ even with 20 UEs.

The LTE D2D communication is based on two physical channels: physical sidelink shared channel (PSSCH) and physical sidelink control channel (PSCCH). PSSCH carries the user plane data, while the PSCCH carries sidelink information (SCI) messages that are used to detect and decode the PSSCH at the receiving device. Each channel uses a combination of resource blocks (RB) in the frequency domain and a set of subframes in the time domain. The PSCCH periods in the time domain are repeated periodically. The device selects the PSCCH/PSSCH resources by itself in Mode 2 from the subframe pool containing all available subframes. The PSCCH channel is defined by a PSCCH subframe bitmap. The PSSCH subframes are defined in time repetition pattern (TRP) bitmap that is included in the SCI message. Each PSCCH transmission is sent twice in different RBs within the same period. If both of these transmissions overlap with another device's PSCCH transmission, neither of them can be received by any devices, which causes delay in the communication since the corresponding PSSCH data transmission cannot be received then either. Colliding or interfering PSSCH transmissions may also reduce the system performance, but they can possibly be solved by hybrid automatic repeat request (HARQ) process, i.e., each message transmission is repeated $\mathrm{n}$ times. Thus, the dimensioning of the resource pools has a significant impact on the performance. NIST has developed an analytical model to determine the best configuration to obtain a target performance level while minimizing the resource pool size [24]. However, it only optimizes the PSCCH. PSSCH optimization is still needed to maximize the sidelink throughput. Since the 802.11p WAVE/DSRC performance was limited only by the coverage area, we ran another test with nodes closer to each other to see how many nodes can be supported within the coverage area so that the delay stays less than $50 \mathrm{~ms}$ and PDR greater than or equal to $95 \%$. We used a CAM message size of 100 bytes. As shown in Figs. 14 and 15, the PDR drops below 95\% with more than 130 nodes, while the delay keeps well below 50 ms with even 250 nodes (PDR has dropped to 60\% then, however).

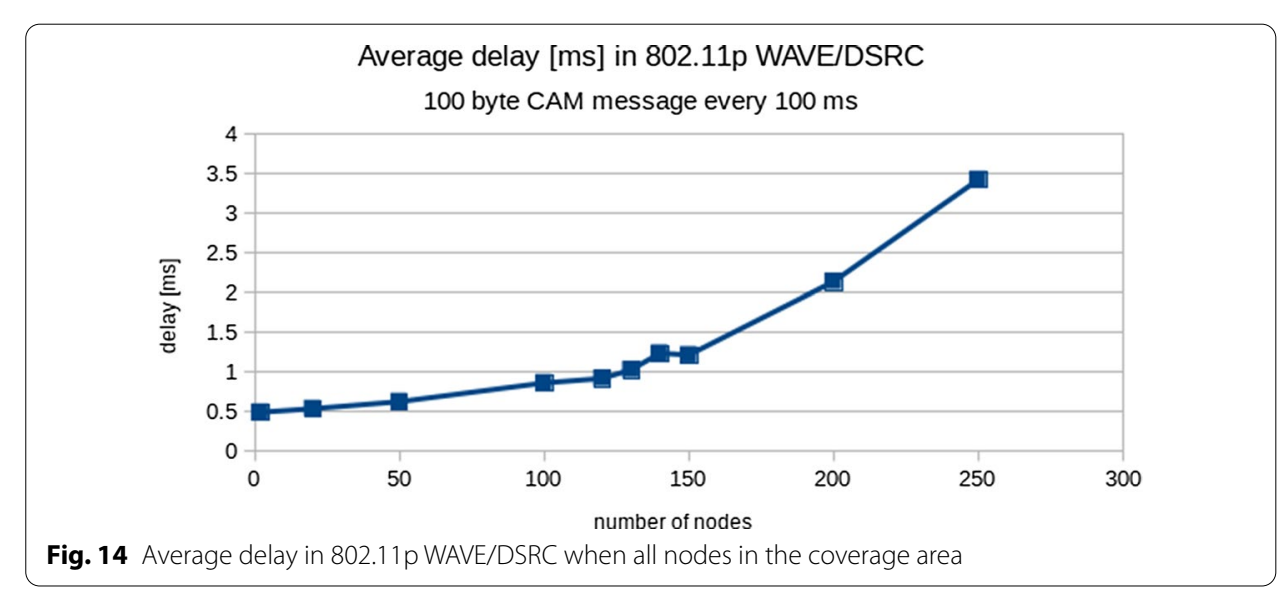


Packet Delivery Ratio in 802.11p WAVE/DSRC

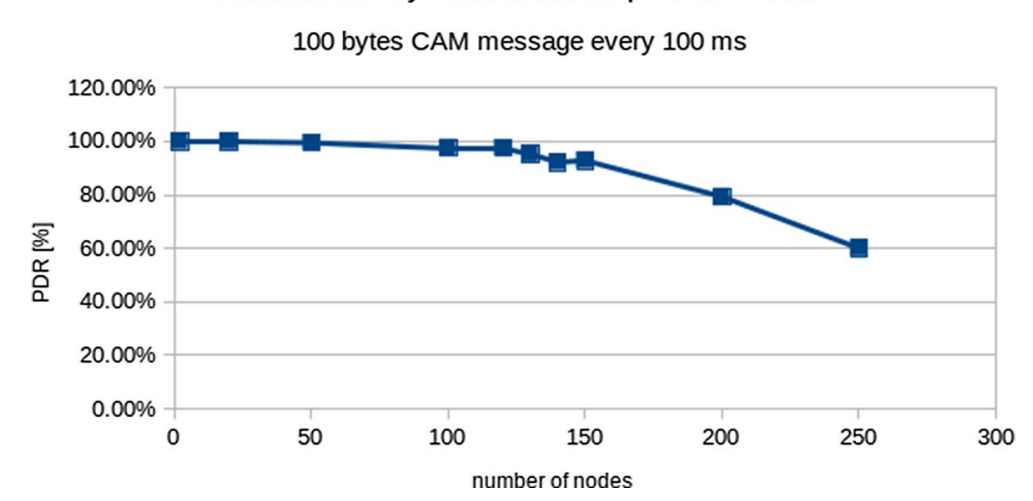

Fig. 15 PDR in 802.11 p WAVE/DSRC when all nodes in the coverage area

\section{Proof-of-concept implementation}

In addition to simulations, it is important to obtain practical experiences of implementing advanced road safety service concepts using actual test networks and vehicles. For this, we implemented a proof of concept of the 5G-assisted automated driving scenario that is introduced in Sect. 4. This section describes the proof-of-concept implementation, which was used in an experimental evaluation for achieving first results and more realistic view of the defined system.

\subsection{Overall system design}

Our proof-of-concept implementation follows the architectural design and publish/ subscribe type of a communication framework originally defined in the paper [1]. The architecture and modular design of the communication framework offer a flexible way of adding and removing software modules depending on the service and test case requirements without a need for redesign or major re-implementation. Consequently, we were able to integrate an existing algorithm implementation into the communication framework for an initial prototype experimentation.

The main design of the prototype is shown in Fig. 16. The obstacle detection algorithm is an application running in the MEC server that can, in general, send warnings to nearby vehicles about obstacles on the road. The algorithm is running in the MEC server as a Docker container. A vehicle equipped with LiDAR measurement tool is sending LiDAR data via TCP to an Apache Kafka information broker located in the MEC server. The algorithm serves as an Apache Kafka subscriber for the LiDAR data, which is sent in point cloud data (PCD) format. Once the algorithm detects an obstacle, it sends a warning message via the MQTT interface to an MQTT broker located in the MEC server. The automated vehicle subscribes to the topic related to the warning and receives the message. The warning message generated by the algorithm gives information on the current time, GPS longitude, GPS latitude, type of warning, and level of warning. The warning message has the following format as string type:

- Epoch time;GPS latitude;GPS longitude;type;level 


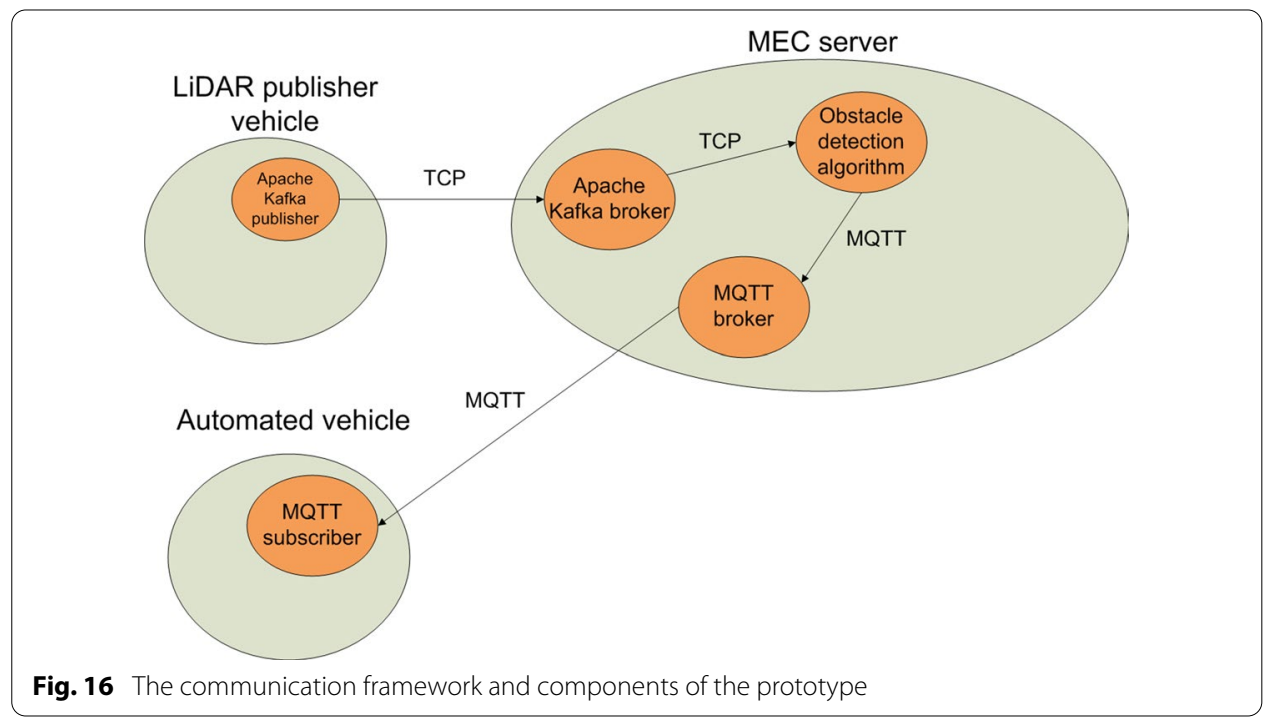

where type is the type of obstacle: either a hard ridge of snow on the road or a physical obstacle. Level is a float value from 0 to 3 , and it informs how large the obstacle is. The higher the value, the larger the obstacle. For implementing the information brokers, we chose Mosquitto [25] as the MQTT broker, while for Apache Kafka, the Confluent software [15] was used.

\subsection{LiDAR data generation and processing components}

In this work, we used a Velodyne VLP-16 sensor. One of its important features is a $360^{\circ}$ - horizontal field of view, which provides a full view of the surrounding scene. However, its vertical resolution is notably coarser. The Velodyne VLP-16 can only obtain readings from 16 lines, vertically distributed within range from $-15^{\circ}$ to $15^{\circ}$.

\subsubsection{Algorithms}

Main processing flow of the software should detect, count and classify vehicles observed by the LiDAR sensor. We developed a multilayered processing pipeline, where input comes from the raw sensor readings and in the output vehicle crossing events are recorded and reported. This pipeline can be roughly divided into the following stages: pre-filtering (outlier removal), clusterization (object detection), cluster tracking and cluster classification.

\subsubsection{Pre-filtering}

As the raw point cloud from the LiDAR sensor usually contains only a small fraction of useful points, some filtering technique should be applied to remove unwanted and irrelevant data points. In this work, we removed points from the back of the LiDAR sensor and ground points. 


\subsubsection{Clusterization}

For the clusterization step, we have implemented hierarchical clustering algorithm, calculated on top of the Minimum Spanning Tree (MST) [26, 27], which is constructed from the filtered point cloud. One of the main algorithmic parameters for MST structure is the maximum length of the edge between two points. Based on that parameter, all possible edges between all points in the remaining point cloud are created. Edges are then sorted in ascending order and added to the tree structure with the greedy algorithm, which starts from the smallest edge and adds next smallest one after another avoiding those edges. This causes loops in the generated structure.

\subsubsection{Static background removal}

Another important aspect is the presence of multiple static elements in the real-time scene. These could be traffic signs and traffic lights, pavements, and the road itself. Each such element can reflect some of the LiDAR points, which are meaningless for obstacle detection.

\subsubsection{Cluster tracking}

As each LiDAR scan represents real-time situation at one moment in time, their automatic clusterization will usually end up into variable number of clusters, returned with completely irrelevant order each time. Robust tracking of corresponding clusters within the recording timeline is necessary to obtain correctly detected object.

\subsubsection{Cluster dissimilarity measurement}

Each cluster can be, for example, described with its centroid point. Dissimilarity between clusters of different scans can be simply taken as Euclidean distance between consecutive centroids.

\subsection{Automated vehicle components and controls}

In the field test experiments, we used VTT's automated vehicle "Martti" [4] as the test vehicle. For testing, "Martti" is set to drive autonomously using a combination of its own sensors and the warning data it receives from the MEC server. In addition, "Martti" is programmed with a local map of the test site. The local map is used in the route planning of the vehicle.

The vehicle control system consists of three components. A vehicle controller component handles the actuators based on instructions it is given. The instructions come from a route planner component that calculates the fastest route to the destination given by the user. The route planner also receives warning data from the third component, a MQTT obstacle data subscriber. This information is used to either slow down the speed of the vehicle or to calculate a completely new route to the destination.

The vehicle's internal communication is handled by an OpenDDS messaging platform. It provides a flexible way of adding software components to the vehicle without affecting former software. We added a new wrapper to the system that converts the warning data from the MEC server to OpenDDS form that the vehicle's other software components know how to utilize it. The route planner software was also 


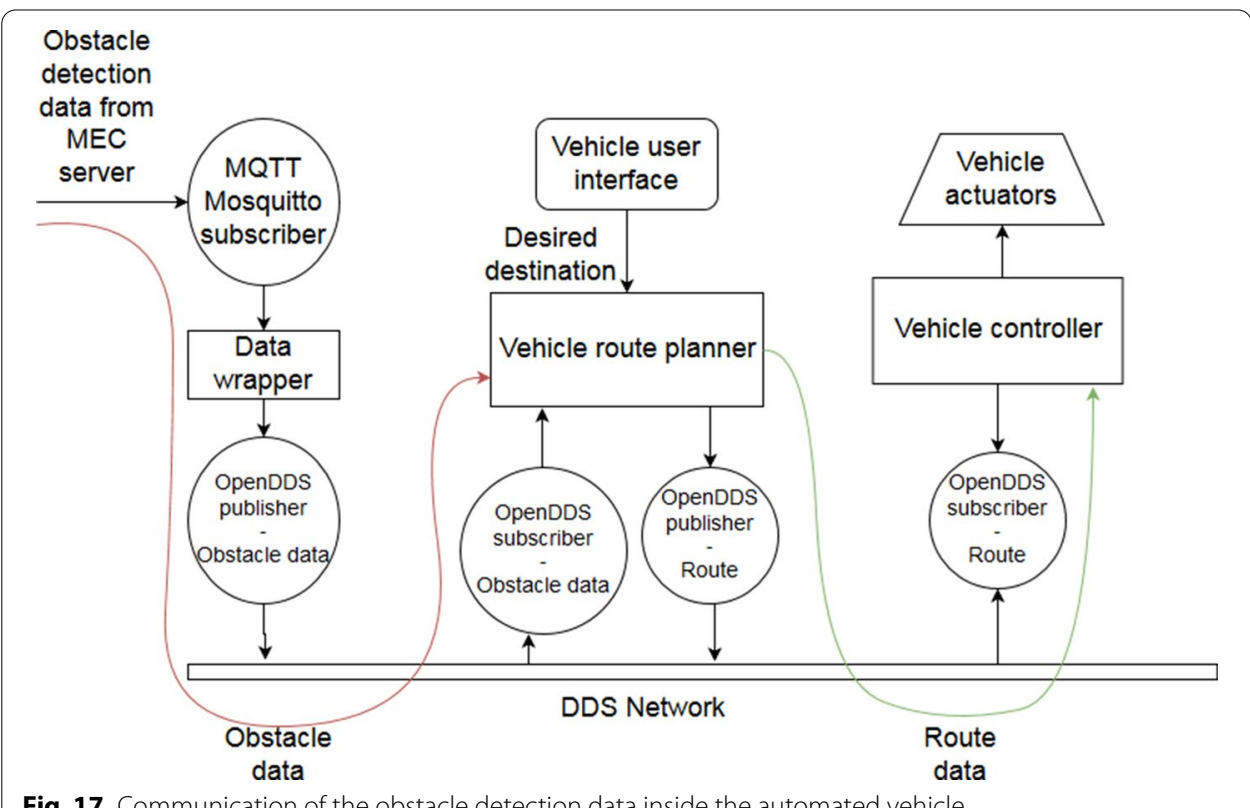

Fig. 17 Communication of the obstacle detection data inside the automated vehicle

redesigned so that it can do recalculations based on the restrictions given by the MEC server. The complete internal data stream from the MEC server to the vehicle actuators is depicted in Fig. 17.

Although the obstacle data can be evaluated in the vehicle, the main driving decisions must be made by the vehicle's internal processing system, e.g., the route planner. This is because all measurements, whether they come from the vehicle itself or from the MEC server can contain errors. If for some reason the vehicle interprets its position incorrectly, all data received from the MEC server are positioned incorrectly in the vehicle's internal map. If driving decisions are based on the incorrect local map, the warning system could increase risks instead of reducing them. In this case, the warnings received from the MEC server are used to increase caution in the warning area or to choose an alternative route. In the future, the vehicles exchanging data could position each other in their own local maps. This would increase the accuracy of warning positions and make the warnings valid for more rapid driving decisions. GPS precision is very important regarding our proposed concept. If GPS precision is deteriorated, the accuracy of the obstacle data coming from the MEC server also declines. It would be beneficial to offer more precise location data for occasions where very high location data precision is required.

\section{Experimental evaluation and results}

The 5G-assisted automated driving prototype was tested in two different environments, namely in a real LTE-A network with outdoor coverage and in an indoor laboratory environment. This section summarizes the test setups and results obtained. 
Table 1 MEC server and client laptop specifications

\begin{tabular}{lll}
\hline & MEC server & Client laptop \\
\hline Model & Dell PowerEdge R510 & HP ZBook \\
Processor & $2{ }^{*}$ Intel Xeon E5606 & 1 * Intel i7-4700MQ \\
Cores (threads) & $4(4) @ 2.13 \mathrm{GHz}$ & $4(8) @ 2.4 \mathrm{GHz}$ \\
Memory & $16 \mathrm{~GB}$ & $8 \mathrm{~GB}$ \\
Operating system & Ubuntu 16.04 & Ubuntu 16.04 \\
\hline
\end{tabular}

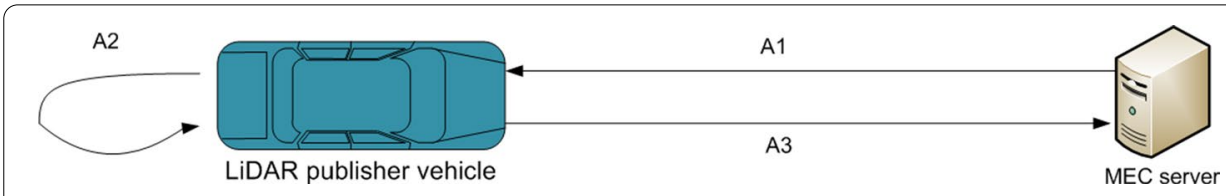

Fig. 18 RTT measurement scenario 1 between the LiDAR publisher vehicle and MEC server

Table 2 RTT measurement results between LiDAR publisher vehicle and MEC server

\begin{tabular}{ll}
\hline Parameter & $\mathrm{A} 1 \rightarrow \mathrm{A} 2 \rightarrow \mathrm{A} 3$ \\
\hline RTT samples & 32098 \\
RTT min & $19.3 \mathrm{~ms}$ \\
RTT max & $487.8 \mathrm{~ms}$ \\
RTT avg & $60.0 \mathrm{~ms}$ \\
RTT stdev & $16.9 \mathrm{~ms}$ \\
\hline
\end{tabular}

\subsection{Field test}

We conducted an experimental evaluation in a winter vehicle test track located in Sodankylä, Finland. More information, including a map of the test track, can be found from [28]. We used two vehicles in the field test, one equipped with LiDAR sensor capability and acting as the LiDAR publisher vehicle, and the second one a real automated car "Martti" [4]. Both vehicles were connected to the 5G test network (5GTN) [29] via a LTE-A connection. The MEC server was hosted by the 5 GTN service core where MEC is realized by following the ETSI distributed EPC approach where MEC is located in the same network as the LTE EPC entities. The MEC server specifications can be found in Table 1.

With the help of the $5 \mathrm{GTN}$ operator, we were able to reserve all LTE radio resources and dedicated network resources. This ensured that there were no other users interfering in the network during the tests. In the field test, we executed network delay measurements by the use of tcpdump, and for analyzing the results, we used the simple tcptrace application.

The main metrics considered in the tests were the round-trip time (RTT) between the LiDAR publisher vehicle and MEC server and RTT between the MEC server and automated vehicle. Figure 18 depicts the measurement scenario for the first test case. The tcptrace application provides $\mathrm{RTT}$ values for route $\mathrm{A} 1 \rightarrow \mathrm{A} 2 \rightarrow \mathrm{A} 3$. This route includes sending packets from the MEC server to the LiDAR publisher vehicle (A1), packet 


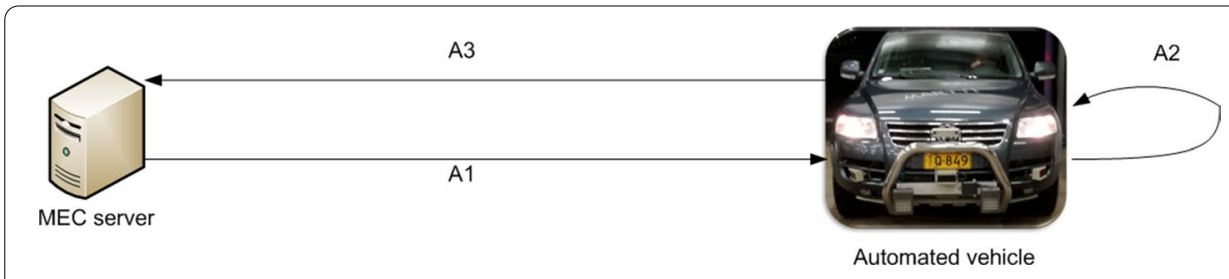

Fig. 19 RTT measurement scenario 2 between MEC server and automated vehicle

Table 3 RTT measurement results between MEC server and automated vehicle

\begin{tabular}{ll}
\hline Parameter & $\mathrm{A} 1 \rightarrow \mathrm{A} 2 \rightarrow \mathrm{A} 3$ \\
\hline RTT samples & 1754 \\
RTT min & $21.0 \mathrm{~ms}$ \\
RTT max & $83.0 \mathrm{~ms}$ \\
RTT avg & $50.7 \mathrm{~ms}$ \\
RTT stdev & $12.4 \mathrm{~ms}$ \\
\hline
\end{tabular}

processing at the LiDAR publisher vehicle (A2), and sending packets from the LiDAR publisher vehicle to the MEC server (A3).

In Table 2, we can see the results from the experiments. A1 $\rightarrow \mathrm{A} 2 \rightarrow \mathrm{A} 3$ is the RTT from the MEC server to the LiDAR publisher vehicle. The minimum RTT is $19.3 \mathrm{~ms}$, maximum $487.8 \mathrm{~ms}$ and the average value is $60.0 \mathrm{~ms}$. To estimate the network delay between the LiDAR publisher vehicle and the MEC server, the RTT values for $\mathrm{A} 1 \rightarrow \mathrm{A} 2 \rightarrow \mathrm{A} 3$ can be divided by two. However, we do not know the processing time A2, but it is assumed to negligible, since the MEC server is only sending ACK messages that have a very low processing time. A reasonable assumption is that the network delay is between 9.65 and $243.9 \mathrm{~ms}$.

Figure 19 depicts the measurement scenario between the MEC server and the automated vehicle. Similar to the previous scenario, A1 $\rightarrow$ A2 $\rightarrow$ A3 is the RTT from the MEC server to the automated vehicle. The results for the aforementioned scenario are shown in Table 3. RTT between the MEC server and the automated vehicle is at minimum $21.0 \mathrm{~ms}$, maximum $83.0 \mathrm{~ms}$ and on average $50.7 \mathrm{~ms}$. As in the first scenario, the packet processing delay at the automated vehicle (A2) is not known. We can, however, reasonably assume that the network delay between the MEC server and the automated vehicle is between 10.5 and $41.5 \mathrm{~ms}$.

\subsection{Laboratory test}

We also conducted test setup in a laboratory environment, as illustrated in Fig. 20. Test setup was realized with the same 5GTN operator, and it used the same MEC resources as in outdoor field tests. In the laboratory test, we got similar dedicated resources for LTE radio connectivity as in the outdoor test to ensure a stable test environment. The test consisted of a MEC server and two client laptops. One client served as a LiDAR publisher and the other as a warning message subscriber that models an automated vehicle. The specifications of the laptops and the MEC server can 


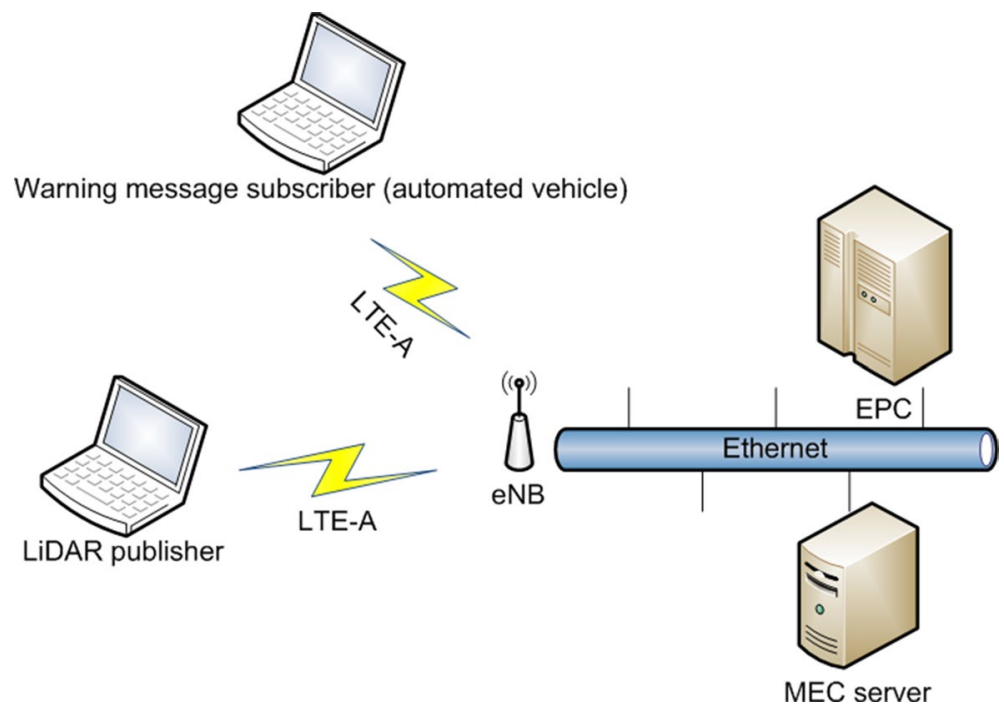

Fig. 20 The testbed used for the laboratory measurements

Table 4 Summary of the RTT measurement results between LiDAR publisher and MEC server

\begin{tabular}{lc}
\hline Parameter & Value \\
\hline RTT samples & 705939 \\
RTT minimum & $14 \mathrm{~ms}$ \\
RTT maximum & $241 \mathrm{~ms}$ \\
RTT average & $45.6 \mathrm{~ms}$ \\
RTT standard deviation & $20.1 \mathrm{~ms}$ \\
\hline
\end{tabular}

Table 5 Summary of the RTT measurement results between MEC server and warning message subscriber

\begin{tabular}{lc}
\hline Parameter & Value \\
\hline RTT samples & 3130 \\
RTT minimum & $12 \mathrm{~ms}$ \\
RTT maximum & $111 \mathrm{~ms}$ \\
RTT average & $47.5 \mathrm{~ms}$ \\
RTT standard deviation & $20.5 \mathrm{~ms}$ \\
\hline
\end{tabular}

be found in Table 1. The clients were connected to the 5GTN [29] via LTE-A using a broadband USB stick. The MEC server was located in the same network as the LTE evolved packet and service core (EPC). In the laboratory tests, we considered the same metrics as in the pilot scenario, the RTT between the LiDAR publisher and the MEC server and the RTT between the MEC server and the warning message subscriber. We also took into account the delay for sending the LiDAR data as an PCD file and the obstacle detection algorithm processing time. We executed twenty runs with each run lasting approximately one minute. 
Table 6 Summary of the PCD file delay measurement results between LiDAR publisher and MEC server

\begin{tabular}{ll}
\hline Parameter & Value \\
\hline Samples & 4871 \\
Minimum & $78.3 \mathrm{~ms}$ \\
Maximum & $2.98 \mathrm{~s}$ \\
Average & $245.9 \mathrm{~ms}$ \\
Standard deviation & $264.6 \mathrm{~ms}$ \\
\hline
\end{tabular}

Table 7 Summary of the obstacle detection algorithm processing delay measurements

\begin{tabular}{ll}
\hline Parameter & Value \\
\hline Samples & 38 \\
Minimum & $80.6 \mathrm{~ms}$ \\
Maximum & $913.6 \mathrm{~ms}$ \\
Average & $544.6 \mathrm{~ms}$ \\
Standard deviation & $219.5 \mathrm{~ms}$ \\
\hline
\end{tabular}

Table 8 Throughput by the LiDAR publisher per experiment

\begin{tabular}{ll}
\hline Parameter & Value \\
\hline Samples & 20 \\
Minimum & $8869624 \mathrm{bps}$ \\
Maximum & $19179912 \mathrm{bps}$ \\
Average & $16428317.2 \mathrm{bps}$ \\
Standard deviation & $2507078.506 \mathrm{bps}$
\end{tabular}

Table 4 shows the summary of the RTT measurement results between the LiDAR publisher and the MEC server. The results are similar to the ones obtained from the pilot scenario. The RTT is on average $45.6 \mathrm{~ms}$, and the maximum and minimum values are $241 \mathrm{~ms}$ and $14 \mathrm{~ms}$, respectively. There is a lot of variation on the results as the minimum and maximum values are far away from each other.

A summary of the RTT results between MEC server and warning message subscriber is presented in Table 5. The minimum value is $12 \mathrm{~ms}$, the maximum value is $111 \mathrm{~ms}$ and the RTT is on average $47.5 \mathrm{~ms}$. The PCD file delay measurement results between LiDAR publisher and MEC server are summarized in Table 6. The results show that there is a very large variation with the PCD file delay. The delay is at minimum $78.3 \mathrm{~ms}$, at maximum $2.98 \mathrm{~s}$ and on average $245.9 \mathrm{~ms}$.

The obstacle detection algorithm processing delay measurements are summarized in Table 7. The processing delay was on average $544.6 \mathrm{~ms}$, at maximum $913.6 \mathrm{~ms}$ and at minimum $80.6 \mathrm{~ms}$. Table 8 presents the throughput by the LiDAR publisher to the MEC server. The table shows that when the data processing is done at the MEC, there needs to be a lot of data for the application to generate warnings or actions.

The approximate delay when an obstacle is detected and when the warning message subscriber receives the warning is generally a sum of the PCD file delay, the algorithm 
processing delay and the network delay between the MEC server and the warning message subscriber. Taking into account the average values in each measurement, the total delay is approximately $245.9 \mathrm{~ms}+544.6 \mathrm{~ms}+23.75 \mathrm{~ms}=814.25 \mathrm{~ms}$ on average, which is close to 1 second. However, as the maximum values for all metrics indicate, the total delay can reach several seconds. The most significant metrics for the total delay are the PCD file delay and the algorithm processing delay. Regarding the radio link, the targeted latency of $5 \mathrm{G}$ is approximately $1 \mathrm{~ms}$, indicating a roughly $30-50 \mathrm{~ms}$ reduction compared to LTE [30]. We could reduce the amount of data transmitted to the network by processing the data at the connected vehicle and sending warning messages to the MEC that could distribute them to nearby vehicles. Consequently, real-time MEC applications for connected vehicles require the use of $5 \mathrm{G}$ networks and efficient hardware in the MEC server as the applications require very low latency, such as $10 \mathrm{~ms}$ [30].

\section{Discussion}

The simulation studies showed the possibility to decrease the end-to-end delay from UE to remote server in the cloud by using MEC. Also, 5G mmWave decreases the delay significantly compared to 4G LTE. In addition, 5G provides larger bandwidth than LTE and enables transmission of, for example, LiDAR data over a $5 \mathrm{G}$ radio. Finally, we studied the delay and packet loss in D2D communication, that is, without any MEC or cloud servers, by using 802.11p and LTE D2D Mode 2-based communication technologies. 802.11p performed better due to the much larger bandwidth compared to what we used for LTE D2D. However, a comparison to LTE D2D Mode 4 was left as a future work item due to the lack of a publicly available simulation model for that at the time of doing the work.

For the proof of concept, the first major milestone was achieved when the field trial was realized and all implemented functionalities worked together in a real operator grade LTE-A test network and with real cars. The implementation and experiments conducted in the winter vehicle test track were also verified by additional tests in a laboratory environment. The test cases and results presented in this paper are very initial ones, and the development and testing will be continued in future work. During the tests, described in this paper, all the available network resources were allocated for the devices used in the test case. The first results proved that it is possible to realize the scenario in real cellular networks. Anyhow, when scaling the scenario for supporting hundreds of connected cars, it is obvious that data filtering and processing may be needed also at the source for avoiding unnecessary flooding of the networks. Edge computing provides a way to minimize the delays and offers extra resources for data processing when needed.

Our future work includes a comparison of different scenarios, such as data processing at the vehicle, data processing at the MEC, and data processing in the cloud in order to find an optimal combination for different services and scenarios. We are also planning to use more computing power with GPU computing capable hardware in the MEC to minimize the processing times and to continue the tests with an actual 5G New Radio connectivity once it is supported by our testbed. 


\title{
9 Conclusion
}

This paper presented a feasibility study of a 5G-inspired road condition warning application running at the mobile network edge. We described the detailed scenario associated with the proposed application as well as its relation to a more generic system architecture specified our in prior work. In terms of practical experimentation, we first evaluated different connectivity and computing options associated with the proposed V2X communication system using ns-3 simulations. Then, we introduced a proof-of-concept implementation of the LiDAR-based obstacle warning scenario together with first results from an experimental evaluation, conducted both in a real vehicle testbed environment and in a laboratory setting. As a result, we obtained the first insights on the feasibility of the overall solution and further enhancements needed. The work will be continued in terms or evaluating the services and solutions with 5G New Radio connectivity as well as analyzing the distribution of the vehicular data processing in more complex scenarios encompassing the vehicle, edge and cloud.

\begin{abstract}
Abbreviations
5GTN: 5 G test network; C-V2X: Cellular vehicle-to-everything communication; CAM: Cooperative Awareness Message; D2D: Device-to-device communication; DENM: Decentralized Environmental Notification Message; DSRC: Dedicated short-range communications; EPC: Evolved packet core; loT: Internet of Things; ITS: Intelligent transport system; LiDAR: Light detection and ranging; LTE: Long-term evolution; LTE-A: LTE advanced; MEC: Multi-access edge computing; MQTT: Message queuing telemetry transport; NAT: Network address translation; PCD: Point cloud data; PDR: Packet delivery ratio; PGW: Packet gateway; PSSCH: Physical sidelink shared channel; PSCCH: Physical sidelink control channel; RAT: Radio access technology; RTT: Round-trip time; V2l: Vehicle-to-infrastructure communication; V2N: Vehicle-to-network communication; V2V:Vehicle-to-vehicle communication; V2X:Vehicle-to-everything communication.
\end{abstract}

\section{Acknowledgements}

The work was conducted in the Challenge Finland 5G-SAFE and Celtic-Next 5G-SAFE-Plus projects, partially funded by Business Finland. The authors would like to thank for the support and for the collaboration with the partners in the project.

\section{Authors' contributions}

TO contributed to the overall storyline and especially to the introduction and motivation of the paper, related work, and the scenario and system design for $5 \mathrm{G}$-assisted automated driving. JM, OM and OM were the main contributors of the proof-of-concept implementation and the corresponding experimental evaluation based on the work published in the EuCNC 2019 paper [5]. MM was the main contributor of the simulation study and findings. JV was the main contributor focused on the LiDAR data generation and processing in the context of the proof-of-concept implementation. All authors read and approved the final manuscript.

\section{Availability of data and materials}

Data are shared only according to the agreements associated with the projects providing funding to the described work. Some of the ns-3 models developed for the simulations have been made public by the authors.

\section{Declarations}

Competing interests

The authors declare that they have no competing interests.

Author details

${ }^{1}$ VTT Technical Research Centre of Finland Ltd., Kaitoväylä 1, 90571 Oulu, Finland. ${ }^{2}$ Unikie Ltd., Elektroniikkatie 3, 90590 Oulu, Finland.

Received: 30 December 2019 Accepted: 30 March 2021

Published online: 16 April 2021

\section{References}

1. T. Ojanperä, J. Mäkelä, O. Mämmelä, M. Majanen, O. Martikainen, Use cases and communications architecture for 5G-enabled road safety services, in 2018 European Conference on Networks and Communications (EuCNC) (2018)

2. 5G PPP Architecture WG: 5G Architecture White Paper Revision 2.0 (online). https://5g-ppp.eu/white-papers/ (2017)

3. ETSI: Mobile Edge Computing (MEC): Framework and Reference Architecture. ETSI GS MEC 003 V1.1.1 (2016-03) (2016) 
4. J. Scholliers, M. Kutila, A. Virtanen, P. Pyykönen, Development of an automated vehicle as an innovation platform, in 25th ITS World Congress (2018)

5. O. Mämmelä, T. Ojanperä, J. Mäkelä, O. Martikainen, J. Väisänen, Evaluation of LiDAR data processing at the mobile network edge for connected vehicles, in 2019 European Conference on Networks and Communications (EUCNC) (2019)

6. IEEE: IEEE Standard for Information Technology_Local and metropolitan area networks_Part 11, Amendment 6: Wireless Access in Vehicular Environments (2010). IEEE Std. 802.11 p-2010 (2010)

7. T. Taleb, K. Samdanis, B. Mada, H. Flinck, S. Dutta, D. Sabella, On multi-access edge computing: a survey of the emerging 5 G network edge cloud architecture and orchestration. IEEE Commun. Surv. Tutor. 19(3), 1657-1681 (2017)

8. ETSI Whitepaper No.23: Cloud RAN and MEC: A Perfect Pairing (2018)

9. M. Emara, M.C. Filippou, D. Sabella, MEC-assisted end-to-end latency evaluations for C-V2X communications, in 2018 European Conference on Networks and Communications (EuCNC), pp. 1-9 (2018)

10. K. Sasaki, N. Suzuki, S. Makido, A. Nakao, Vehicle control system coordinated between cloud and mobile edge computing, in 2016 55th Annual Conference of the Society of Instrument and Control Engineers of Japan (SICE), pp. 1122-1127 (2016)

11. A. Aissioui, A. Ksentini, A.M. Gueroui, T. Taleb, On enabling $5 \mathrm{G}$ automotive systems using follow me edge-cloud concept. IEEE Trans. Veh. Technol. 67(6), 5302-5316 (2018)

12. L. Gillam, K. Katsaros, M. Dianati, A. Mouzakitis, Exploring edges for connected and autonomous driving, in IEEE INFOCOM 2018-IEEE Conference on Computer Communications Workshops (INFOCOM WKSHPS), pp. 148-153 (2018)

13. A. Ogawa, S. Kuroda, H.Y. Katsunori Ushida, T. Kantou, R. Kudo, K. Tateishi, Field experiments on sensor data transmission for 5g-based vehicle-infrastructure cooperation, in VTC2018-Fall (2018)

14. Nokia: Nokia and KDDI successfully trial 4G LTE connected vehicle applications in Japan (online).https://www.nokia. com/en_int/news/releases/2018/04/16/nokia-and-kddi-successfully-trial-4g-Ite-connected-vehicle-applicatio ns-in-japan/

15. Confluent: Confluent Open Source: A developer-optimized distribution of Apache Kafka (online). https://www.confl uent.io/product/confluent-open-source/

16. International Organization for Standardization (ISO): Information technology-Message Queuing Telemetry Transport (MQTT) v3.1.1 (online). https://www.iso.org/standard/69466.html

17. Z. Shelby, K. Hartke, C. Bormann, The Constrained Application Protocol (CoAP). RFC 7252 (2014)

18. T. Ojanperä, T. Sukuvaara, M. Kutila, O. Huuskonen, K. Mäenpää, J. Scholliers, P. Pyykönen, Development and piloting of novel $5 \mathrm{~g}$ enabledroad safety services, in IEEE WCNC 2019 Workshop: Advanced 5 G Radio Access Network Features and Performance (2019)

19. M. Majanen: MEC model for ns-3 (ns3-MEC) (online). https://github.com/mmajanen/ns3-MEC (2018)

20. R. Rouil, A.B.M. F. Cintron, Gamboa, S.: Implementation and validation of an lte d2d model for ns-3, in Workshop on Ns-3 (WNS3 2017) (2017)

21. A. Nabil, K.K.C.D. V. Marojevic: Performance analysis of sensing-based semi-persistent scheduling in c-v2x networks, in CoRR, Abs/1804.10788 (2018)

22. M. Gonzalez-Martin, R.M.-M.J.G. M. Sepulcre: System level evaluation of Ite-v2v mode 4 communications and its distributed scheduling, in IEEE 85th Vehicular Technology Conference (VTC Spring) (2017)

23. F. Eckermann, C.W. J. Freudenthal: Performance analysis of unsupervised Ite device-to-device (d2d) communication, in IEEE Vehicular Technology Conference (VTC-Fall) (2018)

24. D.W. Griffith, R.A.R. F.J. Cintron: Physical sidelink control channel (pscch) in mode 2: performance analysis, in IEEE International Conference on Communications (ICC) (2017)

25. R.A. Light, Mosquitto: server and client implementation of the MQTT protocol. J. Open Sour. Softw. 2(13), 265 (2017)

26. O. Grygorash, Y. Zhou, Z. Jorgensen, Minimum spanning tree based clustering algorithms, in 18th IEEE International Conference on Tools with Artificial Intelligence (ICTAI), pp. 73-81 (2006)

27. C. Zhong, D. Miao, P. Fränti, Minimum spanning tree based split-and-merge: a hierarchical clustering method. Inf. Sci. 181(16), 3397-3410 (2011)

28. Sod5G: Project website (online). http://sod5g.fmi.fi/ (2018)

29. M. Latva-aho, A. Pouttu, A. Hekkala, I. Harjula, J. Mäkelä, Small cell based 5G test network (5GTN), in 2015 International Symposium on Wireless Communication Systems (ISWCS), pp. 231-235 (2015)

30. NGMN Alliance, NGMN 5G White Paper (Technical report, NGMN Alliance, 2015) 